ride of iron diluted with three or four times its weight of water. This restrained the bleeding, but gave some pain. Astringent injections of tannin were used daily, and every third day the speculum was passed, and a sponge saturated with the dilute tincture of perchloride of iron left behind, and subsequently withdrawn. As this gave much distress, injections of the same solution were used alternately with tannin solution (two drachms to the half pint.) The vagina was very sensitive, and all manipulations were attended with distress. But at the end of a month she was free from the bleeding, and in a few weeks more the vagina had resumed its normal state.

CASE 2. Severe chronic vaginitis, with constant and free secretion of pus for over a year; treatment by injections hitherto useless; cured.--This case, though not raw and bleeding like that just described, was with this exception the most severe of any I have seen. Throughout the whole passage it was felt to be granular and velvety, and through the speculum the mucous membrane was observed pouring forth purulent secretion in large quantity. Her age was about thirty, married. The condition had come on gradually, and there was no information leading one to suspect it to have had its origin in gonorrhoa. She had been under treatment by injections for a year before I saw her. She was admitted under my care into Guy's Hospital, and the vagina treated by means of the speculum. Various applications were used in succession: nitrate of silver (twenty grains to the ounce) once; swabbing with the iron solution, as in the former case; plugging with cotton having powdered tannin diffused in it every day for some time-all of which gave gradual improvement. After a fortnight I applied a drachm of anhydrous sulphate of zinc to the upper half of the vagina, plugging it below with cotton well greased. This caused considerable pain and some exfoliation of the vagina; but when it had passed over, the vagina made a quick recovery, aided of course by continued though milder injections, till she left the hospital cured in about a month from admission.

It is not often that cases of like severity are seen; but many present themselves of long standing, with a granular state of the vagina and pus-like discharge, defying the former treatment. But these are obstinate only for the reason that they have not been thoroughly and perseveringly treated; either the syringing of the lotion has been inefficient, applied in vertical posture, or by a sponge only to the vulva, it having never properly reached the upper portion of the vagina. The mode of application is of more importance than the kind of drug employed. The material should be in contact with the diseased membrane for ten minutes at the very least, if possible for an hour, or, better still, for twelve hours; if used as an injection, the patient should be in the horizontal position. But it is best in all cases to commence treatment with a good swabbing of the upper half of the vagina with strong carbolic acid or iodised phenol through the speculum,"which should be retained for five minutes, and then the superfluous portion removed by dry cotton. Care should be taken that a portion be not drawn down to the vulva; if it should be, pain will ensue; this, however, can be removed by washing the part with wet cotton. Care also must be taken lest the carbolic acid run down the speculum on to the thigh or drawers, for if it does, a blister will ensue, and much grumbling likewise. This carbolic application might be repeated in about six or seven days, whilst twice every day an astringent lotion can be employed as strong as can be borne without distress. Of these there are numerous kinds:--Chloralum solution (one ounce to the half pint) tannin (two drachms to the half pint), boroglyceride (on ounce to the half pint), the old sulphate of zine and alum lotion, tannin and alum mixed, tannin with iodoform, bichloride of mercury ( 1 in 1000). Preceding the use of either of these, it is well to employ an antiseptic douche, in order to wash away the secretions which would impede the action of the astringents. But I prefer to apply every three or four days, through the speculum, a stronger astringent than can be employed by the syringe--such as the tincture of muriate of iron diluted three times, stron chloralum; or, as recommended by Dr. Oldham, the walls of the ragina can be kept apart by a plug of cotton. An excellent way of effecting this is by using pledgets of cotton with a string attached, diffusing through them dry powdered tannin and alum with a little iodoform, passing these through the speculum and leaving them behind, and removing them after twelve hours. This can be done every second or third day. But $I$ think that although the healthy vagina tolerates anhydrous sulphate of zinc and the tincture of muriate of iron or the weaker solution, yet when it is abraded or spongy these agents are apt to cause exfoliation of too severe a kind. The ordinary sulphate of zine or alum, however, it will readily bear. These and similar expedients will, if continued steadily, almost always cure the condition as far as the vagina is concerned; but inasmuch as the mucous membrane of the uterus is often involved, attention must be directed to this part, or the vaginitis will recur.

George-street, Hanover-square, W.

\section{MALT AS FOOD.}

\section{Bx J. MILNER FOTHERGILL, M.D.}

MalT as food has a great future before it. In the process of malting (which is a rapid germination) a distinct change is brought about in the seed. By the action of the diastase the insoluble starch is converted into soluble dextrine, which goes sweet in the mouth, so near maltose is it. The malt-combings, which are too rich in albuminoids for the brewer's purposes, are useful when malt is looked at as a food. Malt contains dextrine (some diastase), soluble albuminoids and phosphates, and when ground is an admirable food, while it is not expensive. Its taste is pleasant. It can be made into a pudding with an egg and some milk, and as such is palatable. It can be made a constituent of a milk pudding with advantage. When the brewer mashes his malt with hot water the diastase remaining in the malt converts the dextrine into maltose, which the yeast (next added) breaks up into alcohol and carbonic acid gas. The best white malt is not heated to more than $190^{\circ}$, as the diastase is acted upon injuriously by a heat above that point. Maltose is a sugar which does not readily undergo the acetous fermentation, and, therefore, as Dr. Mitchell Bruce points out in his "Materia Medica and Therapeutics," "will not give rise to acidity and dyspepsia." This is a great matter, as the cane sugar the cook adds to stewed fruit and milk puddings readily undergoes the acetous fermentation in many stomachs. The lævulose sugar of fruit, like maltose, readily undergoes the alcoholic but not the acetous fermentation. Maltose being less powerfully sweet than cane sugar, a greater quantity is necessary to sweeten the pudding. If the raw starch, semolina, sago, or tapioca be first put in the dish by itself, and placed in the oven for an hour (taking care not to lave it burnt by the oven being too hot), not only are the starch cells cracked, but a certain conversion of the starch into dextrine takes place. If to this be then added an equal quantity of ground malt and some hot milk poured on, and the dish be allowed to stand a few minutes before being put into the oven again, the diastase of the malt acts upon the farina and converts it into dextrine and maltose. Dextrine and maltose being soluble, the pudding is very thin. Such a pudding is admirably adapted for invalids and dyspeptics, as requiring scarcely any digestion in the body. For those with whom ordinary milk puddings produce acidity, such a pudding is specially suitable. Ground malt may be added to fresh milk, and forms an admirable food in cases of acute disease. Baked flour perhaps goes better with meat broths, to which it gives a high food value. (Well-baked flour requires but a touch of saliva to render it soluble, and, added to meat broths and gravy soups, renders them very nutritive.) Malt, being sweet, goes better with milk, or apple-water, or tamarındwater, or lemonade, and gives us a food which being all but independent of the digestive act, can be most usefully employed in the sick room. Beef-tea (which alone is scarcely a food) and milk-and-seltzerwater pall upon the palate of the sick person, who craves variety just as do healthy persons. The adoption of ground malt as a food will solve for us one or two knotty questions connected with feeding people when the digestive power is feeble. Drinks like lemonade, made with malt instead of cane-sugar, would not only not go sour in the mouth and stomach, but would contain some phosphates and soluble albuminoirs, and so form admirable beverages in pyrexial states. The many malt extracts now in the market are well adapted for such end. Park-street, W. 